\title{
COVID-19 in Children: Clinical Presentations and Outcomes in Fayoum
} Governorate, Egypt

\author{
Sherin Khamis Hussein ${ }^{1}$, Remon Magdy Yousef ${ }^{1}$, Mohamed Masoud ${ }^{2}$, Mostafa Yehia Abdelwahed ${ }^{3}$ \\ and Rehab Ahmed Mohammed ${ }^{3,{ }^{*}}$ \\ ${ }^{1}$ Pediatric Department, Faculty of Medicine, Fayoum University, Fayoum, Egypt. \\ ${ }^{2}$ Public Health Department, Faculty of Medicine, Fayoum University, Fayoum, Egypt \\ ${ }^{3}$ Physiology Department, Faculty of Medicine, Fayoum University, Fayoum, Egypt \\ "Corresponding author: Physiology Department, Faculty of Medicine, Fayoum University, Fayoum, Egypt. Email: ramo6@fayoum.edu.eg
}

Received 2021 February 13; Revised 2021 June 23; Accepted 2021 July 03.

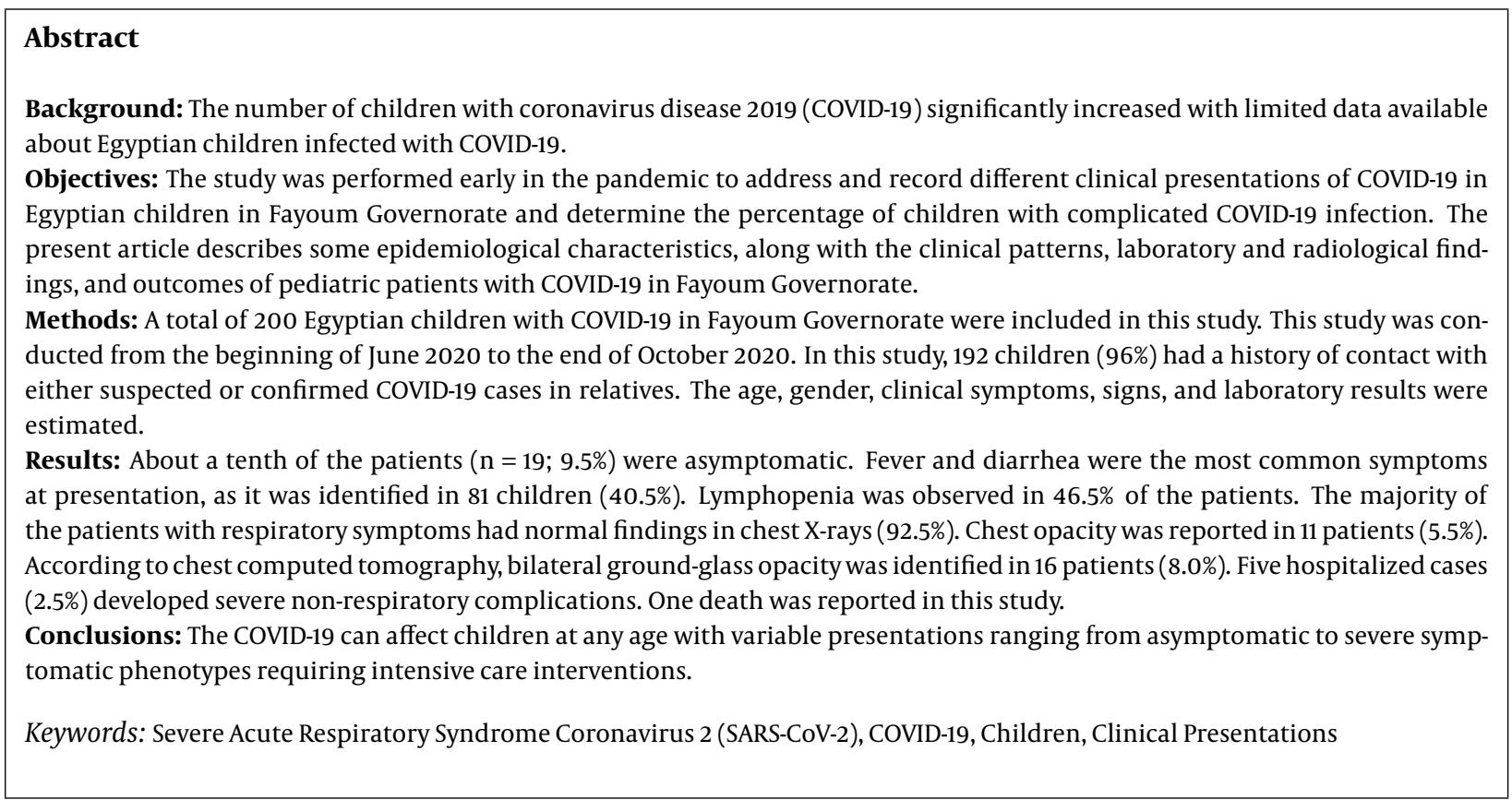

\section{Background}

SARS-CoV-2 is the severe acute respiratory syndrome coronavirus 2 caused by coronavirus disease 2019 (COVID19). Wuhan, China, was the primary place of the infection in December 2019, and then the disease has spread to the world, leading to a cluster of pneumonia cases. A global pandemic in March 2020 was stated by the World Health Organization (1). Most children have fewer symptoms than adults and need no treatment apart from supportive care; however, few of them showed severe complications (2). The SARS-CoV-2 infection may be associated with cytokine storm resulting in acute respiratory distress, macrophage activation syndromes, and immune dysfunction, which is the major cause of mortality associated with COVID-19 (3).
The United States Food and Drug Administration did not approve any drug for the treatment of COVID-19, and no effective safe vaccine is available at the time of data collection (4). Acute COVID-19 in children cannot be easily distinguished from other viral infections based on the symptoms. The differences between pediatric and adult COVID-19 manifestations are due to the changes in both angiotensin-converting enzyme 2 (ACE2) receptors and immune functions; the ACE2 receptors are used by the virus to enter lung cells (5). The variability in the immune responses with age is responsible for different manifestations and complications of the disease in different age groups.

The COVID-19 virus is highly infectious and remains viable on various surfaces from hours to days 6 . The main 
routes of transmission of SARS-CoV-2 are the secretions of the upper respiratory tract, saliva, and aerosol particles, as in coughing and sneezing (6). Viral particles were detected for several weeks in stool after diagnosis, which suspects the fecal-oral transmission of the virus (7).

Children may play a role in viral transmission as they are more common to have upper respiratory tract infections (8). Although few cases of COVID-19 have been diagnosed in children, the majority of those cases have been mild. Most children infected with COVID-19 have either asymptomatic infection or mild illness. Severe illness was reported in $2.5 \%$ of pediatric cases (9). The incubation period of COVID-19 is around 7 days (10), and longer incubation periods were also reported $(11,12)$. The most common manifestations of the disease among children were fever, cough, pharyngeal erythema, diarrhea, vomiting, and fatigue $(13,14)$.

\section{Methods}

This study was conducted as a cross-sectional study. The Research Ethics Committee of Fayoum Medical University, Egypt, approved the study protocol (protocol no.: R 181).

\subsection{Inclusion Criteria}

Children aged from 1 day to 18 years with close contact with polymerase chain reaction (PCR) confirmed adult cases or PCR positive cases for COVID-19 were the inclusion criteria. Moreover, the subjects were completely healthy children before COVID-19 infection.

\subsection{Exclusion Criteria}

Children with no history of close contact with confirmed cases, PCR negative cases for COVID-19, and those with other comorbidities (e.g., diabetic, uremic, and immunocompromised children) were the exclusion criteria.

During the period from the first of June 2020 to the end of October 2020, 200 children from Fayoum, attending Fayoum University Children Hospital, reported as having COVID-19 as they had close contact with confirmed adult cases, whether symptomatic or not, were included in this study. Real-time reverse transcriptase-polymerase chain reaction (RT-PCR) was performed on the respiratory samples of the children to confirm SARS-CoV-2 infection. All the children in the study were hospitalized in the quarantine section of the University Hospital (i.e., the protocol early in the pandemic to prevent the spread of SARS-CoV-2 infection).

The patients' complaints were written and carefully and generally examined for fever, jaundice, pallor, pharyngeal erythema, signs of dehydration, organomegaly, and lymphadenopathy. The chest and heart were carefully examined by inspection and auscultation. The hospitalized patients were investigated and followed up until the time of discharge to record complicated cases and detect the outcomes of all cases in the study. Several investigations were performed on the patients, including complete blood count (CBC) to show changes in the total leucocyte count and differential count, hemoglobin content, platelet count, level of C-reactive protein (CRP), liver enzymes (i.e., aspartate transaminase and alanine transaminase) to exclude liver affection, and urea and creatinine to exclude renal affection. D-dimer and serum ferritin were also measured for all patients. Chest X-ray and computed tomography (CT) were also performed on all children with chest symptoms (as a routine at the beginning of the pandemic in Egypt for all cases with chest symptoms). Some cases need further investigation and imaging according to their presentations as bone marrow biopsy in cases presented by pancytopenia and echocardiography for cases presented by picture simulating Kawasaki disease (KD).

The collected data were tabulated and statistically analyzed using SPSS software (statistical computer package; version 22; SPSS Inc., USA). For quantitative data, the median and interquartile range (IQR) were estimated. For qualitative data, the distribution number and percentage were calculated.

\section{Results}

The median age of the study patients was 108 months, ranging from 13 days to 18 years. The IQR range was 48 - 144 months. More than half of the patients were male (male: female ratio reported as 1.11:1). The majority of the children had a history of contact with either suspected or confirmed COVID-19 cases in relatives. The most common source of infection was parents, as it was considered the index case in 154 children (77.0\%). In the remaining eight patients (4.0\%), the source of infection cannot be traced within the family members (Table 1 ).

About a tenth of the patients were asymptomatic. Fever and diarrhea were the most common symptoms at presentation, as identified, followed by fatigue and malaise, abdominal pain, cough, and arthralgia. It was noticed that less frequently observed symptoms were convulsions, weakness, and respiratory distress, as shown in Table 2.

Table 3 shows the laboratory findings on admission and during the follow-up. Lymphopenia was observed in about half of the patients; however, neutropenia was identified in only $1 \%$ of the subjects. The CRP level was above the normal range in the majority of the cases. 


\begin{tabular}{|c|c|}
\hline & Values \\
\hline \multicolumn{2}{|l|}{ Age, $y$} \\
\hline $13-18$ & $108(48-144)$ \\
\hline \multicolumn{2}{|l|}{ Gender } \\
\hline Female & $95(47.5)$ \\
\hline Male & $105(52.5)$ \\
\hline \multicolumn{2}{|l|}{ Source of infection } \\
\hline Parents & $154(77)$ \\
\hline Other relatives & $38(19)$ \\
\hline Cannot be traced within relatives & $8(4)$ \\
\hline
\end{tabular}

${ }^{\mathrm{a}}$ Values are expressed as median (interquartile range) or No. (\%).

\begin{tabular}{|cc|}
\hline Table 2. Presenting Symptoms and Clinical Examination in Descending Manner \\
\hline Presenting Symptoms and Signs & No. $(\%)$ \\
\hline Asymptomatic & $19(9.5)$ \\
\hline Symptomatic & $181(90.5)$ \\
\hline Fever and diarrhea & $81(40.5)$ \\
\hline Fatigue and malaise & $47(23.5)$ \\
\hline Abdominal pain & $23(11.5)$ \\
\hline Cough & $18(9)$ \\
\hline Bone and muscle pain & $3(1.5)$ \\
\hline Convulsions & $2(1)$ \\
\hline Respiratory distress & $2(1)$ \\
\hline Weakness & $2(1)$ \\
\hline Subcutaneous petechiae & $2(1)$ \\
\hline Severe pallor and abdominal distention & $1(0.5)$ \\
\hline No identified signs (including asymptomatic patients) & $100(50)$ \\
\hline Pharyngitis & $47(23.5)$ \\
\hline Abdominal tenderness & $23(11.5)$ \\
\hline Wheezy chest & $18(9.0)$ \\
\hline Pneumonia & $4(2)$ \\
\hline Dehydration & $3(1.5)$ \\
\hline Signs of Kawasaki vasculitis & $2(1)$ \\
\hline Pancytopenia and hepatosplenomegaly & $1(0.5)$ \\
\hline Hypotonic and paralysis & $1(0.5)$ \\
\hline Signs of acute renal failure & $1(0.5)$ \\
\hline & \\
\hline & \\
\hline
\end{tabular}

The majority of the patients had normal findings in the chest X-ray. Chest opacity was reported in 11 patients. According to the chest CT, the majority of the patients had normal findings; however, bilateral ground-glass opacities (GGOs) were identified in 16 patients, as shown in Table 4.

\begin{tabular}{|c|c|}
\hline Variables & Values \\
\hline \multicolumn{2}{|l|}{ Hemoglobin, gm/dL } \\
\hline $6-14$ & $11(10-11)$ \\
\hline \multicolumn{2}{|l|}{ Platelet, $\times$ 1000 $/ \mathrm{L}$} \\
\hline $36-294$ & $200(170-220)$ \\
\hline \multicolumn{2}{|l|}{ White blood cell, $\times$ 1000/L } \\
\hline $2.3-20$ & $5(4-6)$ \\
\hline \multicolumn{2}{|l|}{ Lymphocyte,\% } \\
\hline $4.8-60$ & $19(16-33)$ \\
\hline Normal cases & $106(53)$ \\
\hline Cases with lymphopenia & $93(46.5)$ \\
\hline Cases with lymphocytosis & $1(0.5)$ \\
\hline \multicolumn{2}{|l|}{ Neutrophil } \\
\hline Cases with neutropenia & $2(1.0)$ \\
\hline Normal cases & $94(47.0)$ \\
\hline Cases with neutrophilia & $104(52)$ \\
\hline \multicolumn{2}{|l|}{ C-reactive protein, $\mathrm{mg} / \mathrm{L}$} \\
\hline $3-276$ & $30(16-53)$ \\
\hline $\operatorname{High}(>6)$ & $189(94.5)$ \\
\hline $\operatorname{Normal}(<6)$ & $11(5.5)$ \\
\hline \multicolumn{2}{|l|}{ Blood urea, mg/L } \\
\hline $20-180$ & $25(22-25)$ \\
\hline \multicolumn{2}{|l|}{ Serum creatinine, $\mathrm{mg} / \mathrm{L}$} \\
\hline $0.3-7$ & $0.5(0.5-0.6)$ \\
\hline Cases with normal urea and creatinine & $196(98)$ \\
\hline Cases with elevated urea and creatinine & $4(2)$ \\
\hline \multicolumn{2}{|l|}{ Alanine transaminase } \\
\hline $22-105$ & $30(29.5-33)$ \\
\hline \multicolumn{2}{|l|}{ Alanine transaminase, IU/mL } \\
\hline $25-100$ & $33(31-37)$ \\
\hline $\begin{array}{l}\text { Cases with normal aspartate transaminase and } \\
\text { alanine transaminase levels }\end{array}$ & $198(99)$ \\
\hline $\begin{array}{l}\text { Cases with elevated aspartate transaminase alanine } \\
\text { transaminase levels }\end{array}$ & $2(1)$ \\
\hline \multicolumn{2}{|l|}{ Ferritin, ng/mL } \\
\hline $33-1100$ & $77(60-100)$ \\
\hline \multicolumn{2}{|l|}{ D-dimer, mcg/mL } \\
\hline $0.1-6.5$ & $0.2(0.1-0.2)$ \\
\hline Cases with normal D-dimer & $200(100)$ \\
\hline Cases with elevated serum ferritin level & $5(2.5)$ \\
\hline
\end{tabular}

${ }^{\mathrm{a}}$ Values are expressed as median (interquartile range) or No. (\%).

Among the hospitalized patients, five hospitalized cases developed severe non-respiratory complications (including two cases of $\mathrm{KD}$, one case of pancytopenia, one case of flaccid paralysis, and one case of acute renal failure $[A R F])$. One death was reported in this study (the case of pancytopenia; mortality rate: $0.5 \%$ ). On the other hand, the remaining hospitalized cases were improved and discharged after 14 days of admission. 


\begin{tabular}{lc}
\hline \multicolumn{1}{l}{ Table 4. Radiological Findings of Patients } \\
\hline \\
\hline Chest X-ray & No. $(\%)$ \\
\hline Normal findings & $185(92.5)$ \\
\hline Mild opacity & $11(5.5)$ \\
\hline Increased bronchovascular markings & $4(2)$ \\
\hline Chest computed tomography & \\
\hline Normal findings & $182(91)$ \\
\hline Bilateral ground-glass opacities & $16(8)$ \\
\hline Increased bronchovascular markings & $2(1)$ \\
\hline
\end{tabular}

\section{Discussion}

The COVID-19 infects children of any age group. The current study aimed to detect the variable clinical presentations, complications, and outcomes of COVID-19 among children early at the start of the pandemic in Fayoum Governorate. The virus is very infectious that was proved by the infection of all members of the family. The majority of children showed mild symptoms, such as fever, malaise, myalgia, dry cough, or gut symptoms (e.g., diarrhea and abdominal pain), which improved after 5 - 7 days. However, some children showed prolonged high fever for more than 7 days with no focus.

The respiratory symptoms ranged from mild cough to typical COVID-19 pneumonia. The current study reported four cases with pneumonia, the youngest of whom was a neonate aged 13 days with a history of contact with COVID19 positive parents and showed the typical COVID-19 pneumonia with bilateral GGOs in the chest CT. This study also reported a 9-year old female complaining of high fever and cough with a history of contact with a positive COVID-19 mother. Her chest X-ray showed exaggerated bronchovascular markings. A chest CT showed typical bilateral GGOs. Patients with typical COVID-19 pneumonia improved 15 days after admission (15).

The laboratory investigations of the patients in the current study showed the CBC of most patients with normal hemoglobin levels (11 - $13 \mathrm{gm} / \mathrm{dL}$ ) in few patients and mild anemia (hemoglobin level: $9 \mathrm{gm} / \mathrm{dl}$ ) in most patients. White blood cell (WBC) counts showed the normal number of leukocytes (WBC: $4-11 \times 10^{3}$ ) in most patients; however, the results showed mild leucopenia in few cases (WBC: $3 \times$ $10^{3}$ ), normal neutrophil count in $4 \%$ of the cases, and neutrophilia in $52 \%$ of the cases.

Neutrophilia is associated with the cytokine storm and hyperinflammation pathognomonic to severe COVID-19 and severe acute respiratory syndrome (16). The previous finding is in agreement with the findings of a study per- formed by Huang et al. (17), who observed a significant correlation between elevated leukocyte count and decreased lymphocyte count among patients with severe COVID-19, compared to those reported for mild cases.

The current study data showed that half of the children (53\%) had normal lymphocyte count, and $46.5 \%$ of the children showed lymphopenia. Lymphopenia (lymphocyte less than $20 \%$ of the total $\mathrm{WBC}$ ) is a common finding in patients with COVID-19 infection and may be due to the defective immune response to the virus (18). The previous finding is not in agreement with the findings of a study conducted by Bari et al. (19), who concluded that the blood picture of COVID-19 in children does not show leukopenia and lymphopenia except in cases complicated with multisystem inflammatory syndrome in children (MIS-C).

Blood lymphocyte percentage is the most significant parameter suspecting the disease progressions (20). Normal platelet count was noticed in all cases in the study except those with complications. The liver synthesizes CRP, an acute-phase reactant, and increased inflammatory conditions. CRP increased in $94.5 \%$ of patients with COVID-19 infection. The CRP and absolute lymphocyte count were used to assess the disease progression in patients with COVID-19 infection (21). The aforementioned results are in agreement with the results of a study carried out by Yun et al. (22), whose study revealed that patients with COVID19 showed decreased lymphocyte counts and proportions, decreased eosinophil counts and proportions, and an increase in CRP.

D-dimer as a fibrin degradation product used to measure clot formation was normal in all patients in the present study. In the COVID-19 pandemic, elevated D-dimer levels have been associated with disease severity and mortality trends. Serum ferritin was normal in uncomplicated patients with COVID-19 infection.

A small portion of the patients in the current study developed severe non-respiratory complications. Among the unpredicted presentations of the virus, two female cases reported in this study, with 4 and 10 years of age, were presented with high fever for more than 7 days, rash, tachycardia, severe conjunctivitis, and generalized lymphadenopathy. The CRP and serum ferritin levels of the two patients were very high. The $\mathrm{CBC}$ showed marked leukocytosis and lymphopenia. Liver enzymes were elevated in this study. In addition, cardiac enzymes were normal. Echocardiography was performed to exclude coronary aneurysms showing decreased ejection fraction in both females. Abdominal ultrasound showed multiple enlarged mesenteric lymph nodes in one of the cases and cervical lymphadenopathy in the other. Chest CT was normal for the two patients. The previous clinical picture in COVID19 patients was similar to $\mathrm{KD}$, which is a systemic vasculitis 
affecting children under 5 years of age.

The KD is considered the first cause of acquired heart disease in children (22). The KD diagnosis depends on the clinical features. The administration of intravenous immunoglobulins is the main line of treatment. The previous clinical picture of COVID associated KD should be distinguished from MIS-C or pediatric inflammatory, multisystem syndrome (23). The previous finding is in accordance with the findings of a study performed by Rubens et al. (24), who reported that patients with MIS-C might present with some features consistent with $\mathrm{KD}$ (e.g., fever and mucocutaneous changes); however, MIS-C is a different clinical entity.

There are several points for differences, with the higher incidence of MIS-C patients presenting in older age than KD with increased gastrointestinal and neurological signs, higher incidence of myocarditis and cardiac involvement, increased ferritin, marked leukopenia, lymphopenia, and thrombocytopenia in patient with MIS-C (25). Italy was the first European region with a very high number of SARSCoV2 epidemics after China (26); therefore, Italy showed a high number of severely ill patients with a clinical picture similar to Kawasaki shock syndrome (27). The typical manifestations of this syndrome are persistent high fever, abdominal pain, diarrhea, skin rash (i.e., mucocutaneous involvement), arthralgia, cough, conjunctivitis, periorbital edema, strawberry tongue, and rapidly deteriorating clinical conditions with the signs of dehydration (28).

Furthermore, COVID-19 can affect nervous system. The current study reported a 14-year-old male case, complaining of inability to walk or stand and then dripping of saliva (i.e., bulbar symptoms). These manifestations occurred suddenly 6 days after contact with COVID-19 positive grandfather, grandmother, and aunt. Furthermore, his CBC showed marked lymphopenia with very high CRP, chest CT showed small GGO, and PCR for COVID-19 was positive. The child was mechanically ventilated. He improved after three sessions of plasmapheresis and started weaning from a ventilator. The ascending symmetrical flaccid paralysis, with hyporeflexia with or without cranial nerve involvement, is known as Guillain-Barré syndrome (GBS). The GBS was preceded by respiratory or intestinal infections or vaccinations. The GBS is an immune-mediated disorder. It is believed that COVID-19 enhances the production of antibodies against the specific gangliosides of the peripheral nervous system (29).

The previous finding is in agreement with the findings of a study conducted by Curtis et al. (30), reporting an 8-year-old male patient with ascending progressive weakness and areflexia. The magnetic resonance imaging of the spine revealed the abnormal enhancement of posterior nerve roots consistent with GBS. The results of SARS-CoV-
2 nucleic acid amplification and SARS-CoV-2 immunoglobulin $\mathrm{G}$ antibody tests were positive (30). There are many cases with GBS associated with COVID-19 infection worldwide (31). The neurological symptoms presented 7-10 days after the initial respiratory infection. COVID-19 was diagnosed by RT-PCR.

One of the uncommon presentations of COVID-19 was ARF in one child aged 5 years in the present study. The child was completely normal before infection with COVID-19. He presented to the University Hospital with diarrhea, hematuria, and pallor. The CBC showed marked anemia and thrombocytopenia. Moreover, urea and creatinine were highly elevated with very high CRP. He was admitted to the Emergency Room for a rapid correction of electrolytes and acid-base imbalance. The previous finding is in agreement with the findings of a study performed by Abdalbary et al. (32), who reported kidney infection in many cases with COVID-19 and acute kidney injury associated with higher rates of mortality. The ACE2 is highly expressed in renal tubules leading to tubular damage caused by SARS-CoV-2 (32). Moreover, the virus can induce bone marrow depression and pancytopenia.

The current study reported one male child aged 3 months, presented with severe pallor and hepatosplenomegaly immediately after the COVID-19 infection. His CBC showed red blood cell count ( $\left.1 \mathrm{million} / \mathrm{mm}^{3}\right)$, hemoglobin ( $6.7 \mathrm{gm} / \mathrm{dL}), \mathrm{WBC}$ count (2.3 thousand), and platelet count (39 thousand). The child was referred to Cancer Hospital for children in Cairo 37357 for bone marrow biopsy; however, the case died rapidly. This finding is consistent with the findings of a study carried out by Tiwari et al. (33), who reported a case of pancytopenia induced by COVID-19.

One of the limitations of this study is its small sample size, as the study was performed early in the pandemic.

\subsection{Conclusions}

The COVID-19 virus as a highly infectious one, which affects children at any age with variable presentations ranging from asymptomatic to severe symptomatic phenotypes, needs intensive care interventions. The majority of children can pass without infection; however, a limited number of children showed severe complications raising the morbidity and mortality rates of the disease. Five cases were reported by such presentations.

\section{Footnotes}

Authors' Contribution: Conception and design: Sherin Khamis Hussein, Remon Magdy Yousef, and Mostafa Yehia Abdelwahed. Collection of the cases: Sherin Khamis Hussein, Remon Magdy Yousef, Mostafa Yehia Abdelwahed, and 
Rehab Ahmed Mohammed. Acquisition of the data: Sherin Khamis Hussein, Rehab Ahmed Mohammed, and Mostafa Yehia Abdelwahed. Analysis and interpretation of the data: Mohamed Masoud and Mostafa Yehia Abdelwahed. Drafting the article and revising the data: Mohamed Masoud, Rehab Ahmed Mohammed, and Mostafa Yehia Abdelwahed. Final approval of the manuscript: Mohamed Masoud, Rehab Ahmed Mohammed, and Remon Magdy Yousef.

Conflict of Interests: The authors declare that there is no conflict of interest.

Ethical Approval: The Research Ethics Committee of Fayoum Medical University, Egypt, approved the study protocol (protocol no.: R 181). The patient's consent was not required for this study.

Funding/Support: There was no type of funding for the present study.

\section{References}

1. $\mathrm{Wu} \mathrm{Z}$, McGoogan JM. Characteristics of and Important Lessons From the Coronavirus Disease 2019 (COVID-19) Outbreak in China: Summary of a Report of 72314 Cases From the Chinese Center for Disease Control and Prevention. JAMA. 2020;323(13):1239-42. doi: 10.1001/jama.2020.2648. [PubMed: 32091533].

2. Dhama K, Khan S, Tiwari R, Sircar S, Bhat S, Malik YS, et al. Coronavirus Disease 2019-COVID-19. Clin Microbiol Rev. 2020;33(4). doi: 10.1128/CMR.00028-20. [PubMed: 32580969]. [PubMed Central: PMC7405836].

3. Castagnoli R, Votto M, Licari A, Brambilla I, Bruno R, Perlini S, et al. Severe Acute Respiratory Syndrome Coronavirus 2 (SARS-CoV-2) Infection in Children and Adolescents: A Systematic Review. JAMA Pediatr. 2020;174(9):882-9. doi: 10.1001/jamapediatrics.2020.1467. [PubMed: 32320004].

4. Ortiz-Prado E, Simbana-Rivera K, Gomez-Barreno L, Rubio-Neira M, Guaman LP, Kyriakidis NC, et al. Clinical, molecular, and epidemiological characterization of the SARS-CoV-2 virus and the Coronavirus Disease 2019 (COVID-19), a comprehensive literature review. Diagn Microbiol Infect Dis. 2020;98(1):115094. doi: 10.1016/j.diagmicrobio.2020.115094. [PubMed: 32623267]. [PubMed Central: PMC7260568].

5. Molloy EJ, Bearer CF. COVID-19 in children and altered inflammatory responses. Pediatr Res. 2020;88(3):340-1. doi: 10.1038/s41390-020-0881y. [PubMed: 32244248].

6. Sanche S, Lin YT, Xu C, Romero-Severson E, Hengartner N, Ke R. High Contagiousness and Rapid Spread of Severe Acute Respiratory Syndrome Coronavirus 2. Emerg Infect Dis. 2020;26(7):1470-7. doi: 10.3201/eid2607.200282. [PubMed: 32255761]. [PubMed Central: PMC7323562].

7. Gu J, Han B, Wang J. COVID-19: Gastrointestinal Manifestations and Potential Fecal-Oral Transmission. Gastroenterology. 2020;158(6):1518-9. doi: 10.1053/j.gastro.2020.02.054. [PubMed: 32142785]. [PubMed Central: PMC7130192].

8. She J, Liu L, Liu W. COVID-19 epidemic: Disease characteristics in children. J Med Virol. 2020;92(7):747-54. doi: 10.1002/jmv.25807. [PubMed: 32232980]. [PubMed Central: PMC7228385].

9. Cruz AT, Zeichner SL. COVID-19 in Children: Initial Characterization of the Pediatric Disease. Pediatrics. 2020;145(6). doi: 10.1542/peds.20200834. [PubMed: 32179659].

10. Jutzeler CR, Bourguignon L, Weis CV, Tong B, Wong C, Rieck B, et al. Comorbidities, clinical signs and symptoms, laboratory findings, imag- ing features, treatment strategies, and outcomes in adult and pediatric patients with COVID-19: A systematic review and meta-analysis. Travel Med Infect Dis. 2020;37:101825. doi: 10.1016/j.tmaid.2020.101825. [PubMed: 32763496]. [PubMed Central: PMC7402237].

11. Posfay-Barbe KM, Wagner N, Gauthey M, Moussaoui D, Loevy N, Diana A, et al. COVID-19 in Children and the Dynamics of Infection in Families. Pediatrics. 2020;146(2). doi: 10.1542/peds.2020-1576. [PubMed: 32457213].

12. Kaul D. An overview of coronaviruses including the SARS-2 coronavirus - Molecular biology, epidemiology and clinical implications. Curr Med Res Pract. 2020;10(2):54-64. doi: 10.1016/j.cmrp.2020.04.001. [PubMed: 32363221]. [PubMed Central: PMC7194867].

13. Kan MJ, Grant LMC, Muna MA, Greenhow TL. Fever Without a Source in an Infant Due to Severe Acute Respiratory Syndrome Coronavirus2.J Pediatric Infect Dis Soc. 2021;10(1):49-51. doi: 10.1093/jpids/piaa044. [PubMed: 32318729]. [PubMed Central: PMC7188112].

14. Xia W, Shao J, Guo Y, Peng X, Li Z, Hu D. Clinical and CT features in pediatric patients with COVID-19 infection: Different points from adults. Pediatr Pulmonol. 2020;55(5):1169-74. doi: 10.1002/ppul.24718. [PubMed: 32134205]. [PubMed Central: PMC7168071].

15. Parisi GF, Indolfi C, Decimo F, Leonardi S, Miraglia Del Giudice M. COVID-19 Pneumonia in Children: From Etiology to Management. Front Pediatr. 2020;8:616622. doi: 10.3389/fped.2020.616622. [PubMed: 33381482]. [PubMed Central: PMC7767924].

16. Kim JS, Lee JY, Yang JW, Lee KH, Effenberger M, Szpirt W, et al. Immunopathogenesis and treatment of cytokine storm in COVID19. Theranostics. 2021;11(1):316-29. doi: 10.7150/thno.49713. [PubMed: 33391477]. [PubMed Central: PMC7681075].

17. Huang G, Kovalic AJ, Graber CJ. Prognostic Value of Leukocytosis and Lymphopenia for Coronavirus Disease Severity. Emerg Infect Dis. 2020;26(8):1839-41. doi: 10.3201/eid2608.201160. [PubMed: 32384045]. [PubMed Central: PMC7392413].

18. Lippi G, Plebani M. The critical role of laboratory medicine during coronavirus disease 2019 (COVID-19) and other viral outbreaks. Clin Chem Lab Med. 2020;58(7):1063-9. doi: 10.1515/cclm-2020-0240. [PubMed: 32191623].

19. Bari A, Ch A, Bano I, Saqlain N. Is leukopenia and lymphopenia a characteristic feature of COVID-19 in children? Pak J Med Sci. 2021;37(3):869-73. doi: 10.12669/pjms.37.3.3848. [PubMed: 34104180]. [PubMed Central: PMC8155415].

20. Wang J, Jiang M, Chen X, Montaner LJ. Cytokine storm and leukocyte changes in mild versus severe SARS-CoV-2 infection: Review of 3939 COVID-19 patients in China and emerging pathogenesis and therapy concepts. J Leukoc Biol. 2020;108(1):17-41. doi: 10.1002/JLB.3COVR0520272R. [PubMed: 32534467]. [PubMed Central: PMC7323250].

21. Henry BM, Lippi G, Plebani M. Laboratory abnormalities in children with novel coronavirus disease 2019. Clin Chem Lab Med. 2020;58(7):1135-8. doi: 10.1515/cclm-2020-0272. [PubMed: 32172227].

22. Yun $H$, Sun Z, Wu J, Tang A, Hu M, Xiang Z. Laboratory data analysis of novel coronavirus (COVID-19) screening in 2510 patients. Clin Chim Acta. 2020;507:94-7. doi: 10.1016/j.cca.2020.04.018. [PubMed: 32315614]. [PubMed Central: PMC7166029].

23. Marchesi A, Tarissi de Jacobis I, Rigante D, Rimini A, Malorni W, Corsello G, et al. Kawasaki disease: guidelines of the Italian Society of Pediatrics, part I - definition, epidemiology, etiopathogenesis, clinical expression and management of the acute phase. Ital J Pediatr. 2018;44(1):102. doi: 10.1186/s13052-018-0536-3. [PubMed: 30157897]. [PubMed Central: PMC6116535].

24. Rubens JH, Akindele NP, Tschudy MM, Sick-Samuels AC. Acute covid-19 and multisystem inflammatory syndrome in children. BMJ. 2021;372:n385. doi: 10.1136/bmj.n385. [PubMed: 33648933].

25. Cheung EW, Zachariah P, Gorelik M, Boneparth A, Kernie SG, Orange JS, et al. Multisystem Inflammatory Syndrome Related to COVID-19 in Previously Healthy Children and Adolescents in New York City. JAMA. 2020;324(3):294-6. doi: 10.1001/jama.2020.10374. [PubMed: 32511676]. [PubMed Central: PMC7281352]. 
26. Li Y, Zheng Q, Zou L, Wu J, Guo L, Teng L, et al. Kawasaki disease shock syndrome: clinical characteristics and possible use of IL-6, IL-10 and IFN-gamma as biomarkers for early recognition. Pediatr Rheumatol Online J. 2019;17(1):1. doi: 10.1186/s12969-018-0303-4. [PubMed: 30611297]. [PubMed Central: PMC6321686].

27. Riphagen S, Gomez X, Gonzalez-Martinez C, Wilkinson N, Theocharis P. Hyperinflammatory shock in children during COVID-19 pandemic. Lancet. 2020;395(10237):1607-8. doi: 10.1016/S0140-6736(20)31094-1. [PubMed: 32386565]. [PubMed Central: PMC7204765].

28. Feldstein LR, Rose EB, Horwitz SM, Collins JP, Newhams MM, Son MBF, et al. Multisystem Inflammatory Syndrome in U.S. Children and Adolescents. N Engl J Med. 2020;383(4):334-46. doi: 10.1056/NEJMoa2021680. [PubMed: 32598831]. [PubMed Central: PMC7346765].

29. Toscano G, Palmerini F, Ravaglia S, Ruiz L, Invernizzi P, Cuzzoni MG, et al. Guillain-Barre Syndrome Associated with SARS-CoV-2. N EnglJMed.2020;382(26):2574-6. doi:10.1056/NEJMc2009191. [PubMed:
32302082]. [PubMed Central: PMC7182017].

30. Curtis M, Bhumbra S, Felker MV, Jordan BL, Kim J, Weber M, et al. Guillain-Barre Syndrome in a Child With COVID-19 Infection. Pediatrics. 2021;147(4). doi:10.1542/peds.2020-015115. [PubMed: 33093141].

31. Zhao H, Shen D, Zhou H, Liu J, Chen S. Guillain-Barre syndrome associated with SARS-CoV-2 infection: causality or coincidence? Lancet Neurol. 2020;19(5):383-4. doi: 10.1016/S1474-4422(20)30109-5. [PubMed: 32246917]. [PubMed Central: PMC7176927].

32. Abdalbary M, Sheashaa H. Acute kidney injury in patients with coronavirus disease 2019-how much do we know? J Egypt Soc Nephrol Transplant. 2020;20(4):204.

33. Tiwari N, Krishnan NR, Singh S, Madan J, Nath D, Tripathi S, et al. COVID 19 and Pancytopenia: A Single Case Experience of a Pediatrics Institute. Saudi J Pathol Microbiol. 2020;5(5):308-10. doi: 10.36348/sjpm.2020.v05i05.016. 